\title{
Herzkatheterinterventionen beim Neugeborenen
}

Frank-Thomas Riede, Christian Paech, Ingo Dähnert

\begin{abstract}
Herzfehler sind mit einer Prävalenz von ungefähr 1 \% die häufigsten angeborenen Fehlbildungen [1]. Bei kritischen angeborenen Herzfehlern (kAHF) ist eine rechtzeitige - meist kardiochirurgische - Therapie überlebenswichtig. Herzkatheterinterventionen können beim Neugeborenen dazu beitragen, den präoperativen Zustand und das OP-Ergebnis zu verbessern oder - seltener - eine OP zu vermeiden.
\end{abstract}

\begin{abstract}
Merke
Neugeborene mit kritischen angeborenen Herzfehlern können postnatal oligo- oder asymptomatisch sein.
\end{abstract}

\section{Allgemeine Aspekte}

Ein breites Spektrum von Herzkatheterinterventionen hat mittlerweile einen wichtigen Stellenwert in der Behandlung von Neugeborenen mit angeborenen Herzfehlern. Die wachsende Erfahrung der behandelnden Zentren und die Miniaturisierung des Materials haben dazu geführt, dass auch komplexe Eingriffe mit einem vertretbaren Risiko durchgeführt werden können.

\section{Durchführung der Herzkatheterintervention und Gefäßzugänge}

Herzkatheterinterventionen können in Analgosedierung mit Lokalanästhesie oder - je nach den institutionellen Erfahrungen - in Allgemeinnarkose durchgeführt werden.

Außer den im Kindesalter meist üblichen inguinalen Gefäßzugängen kommen beim Neugeborenen auch die Nabelgefäße in Betracht; seltene Alternativen sind in Abhängigkeit von der geplanten Intervention die A. axillaris oder die A. carotis.

Eine mittels Seldinger-Technik in das Gefäß eingebrachte Schleuse erlaubt den Wechsel zwischen verschiedenen Kathetern. Beim Neugeborenen können viele Eingriffe über Schleusen durchgeführt werden, deren Innendurchmesser 4 French beträgt ( $\triangleright$ Abb. 1). „French“ wird im angloamerikanischen Sprachraum vereinfacht für „Charrière“ verwendet; 3 French entsprechen somit 3 Charrière bzw. $1 \mathrm{~mm}$.

\section{THERAPIE}

Grundsätzliche Optionen von Herzkatheterinterventionen

- Öffnen (Ballons, Perforation)

- Offen halten (Stents)

- Schließen (Coils, Occluder, Plugs)

- Entfernen (Snare-Katheter, Bioptome)

$\begin{array}{ll}\text { ABKÜRZUNGEN } \\ \text { AHF } & \text { angeborener Herzfehler } \\ \text { AK } & \text { Aortenklappe } \\ \text { ASD } & \text { Vorhofseptumdefekt } \\ \text { BAS } & \text { Ballonatrioseptostomie } \\ \text { d-TGA } & \text { dextro-Transposition der großen Arterien } \\ \text { F }_{\mathbf{i}} \text { }_{2} & \text { inspiratorische Sauerstofffraktion } \\ \text { GG } & \text { Geburtsgewicht } \\ \text { ISTA } & \text { Aortenisthmusstenose } \\ \text { kAHF } & \text { kritischer angeborener Herzfehler } \\ \text { LV } & \text { linker Ventrikel } \\ \text { LVEDP } & \text { linksventrikulärer enddiastolischer Druck } \\ \text { LVOTO } & \text { linksventrikuläre Ausflusstraktobstruktion } \\ \text { MI } & \text { Mitralinsuffizienz } \\ \text { MK } & \text { Mitralklappe } \\ \text { PA-IVS } & \text { Pulmonalatresie mit intaktem Ventrikelseptum } \\ \text { PDA } & \text { offener Ductus arteriosus } \\ \text { PFO } & \text { offenes Foramen ovale } \\ \text { PHT } & \text { pulmonale Hypertension } \\ \text { PPHN } & \text { persistierende pulmonale Hypertension des } \\ & \text { Neugeborenen } \\ \text { R/L-Shunt } & \text { Rechts-links-Shunt } \\ \text { RV } & \text { rechter Ventrikel } \\ \text { S } \mathbf{O}_{2} & \text { pulsoxymetrische Sauerstoffsättigung } \\ \text { SSW } & \text { Schwangerschaftswoche } \\ \text { VSD } & \text { Ventrikelseptumdefekt } \\ & \end{array}$




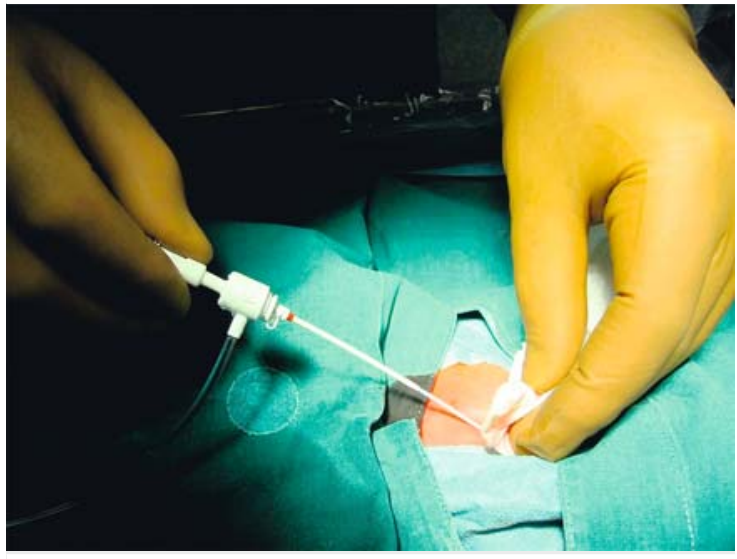

- Abb. 1 Über den nach Punktion eines Femoralgefäßes mit einer Venenkanüle vorgeschobenen Führungsdraht wird eine 4-Fr-Schleuse platziert.

\section{Komplikationen}

Genaue Daten für die Komplikationsrate bei Herzkatheterinterventionen in Deutschland sind nicht bekannt-bislang gibt es für die Behandlung von Patienten mit angeborenen Herzfehlern keine gesetzlich verpflichtende Qualitätssicherung. Viele Zentren beteiligen sich jedoch auf freiwilliger Basis am Projekt „Nationale Qualitätssicherung angeborener Herzfehler“, das gemeinsam von den deutschen Fachgesellschaften für Thorax-, Herz- und Gefäßchirurgie und für Pädiatrische Kardiologie getragen wird. Die Daten werden jährlich ausgewertet und den jeweiligen Zentren mitgeteilt.
Die Rate an Komplikationen bei Herzkatheterinterventionen im Neugeborenenalter ist zwar höher als bei älteren Kindern [2-4], die prozedurbezogene Mortalität ist aber auch in dieser Altersgruppe sehr gering [5-7].

Zu den häufigsten Komplikationen zählen Herzrhythmusstörungen und Gefäßkomplikationen:

- Extrasystolen, (supra-)ventrikuläre Tachykardien, Schenkelblockierungen und AV-Blockierungen $\mathrm{II}^{\circ}-\mathrm{II}^{\circ}$ sistieren meist spontan. Selten ist eine medikamentöse oder elektrische Kardioversion, Defibrillation, temporäre Schrittmacherstimulation oder eine kardiopulmonale Reanimation erforderlich. Bei häufigem Wechsel von Kathetern kann es zu einem transfusionsbedürftigen Blutverlust kommen.

- In mehreren Untersuchungen konnte gezeigt werden, dass die Häufigkeit von Gefäßkomplikationen nach Herzkathetereingriffen im 1. Lebensjahr mit alleiniger klinischer Untersuchung deutlich unterschätzt wird. So lassen sich bei Anwendung einer gezielten Ultraschalldiagnostik arterielle Thrombosen in ungefähr $20-30 \%$ der Fälle nachweisen [8-10]. Allerdings bilden sich davon bis zu $90 \%$ unter Behandlung mit unfraktioniertem oder niedermolekularem Heparin zurück $[11,12]$.

\section{Merke}

Embolien, Perforationen von Herz und Gefäßen sowie Dislokationen von Implantaten sind seltene, aber potenziell schwerwiegende Komplikationen von Herzkatheterinterventionen.

\section{FALLBEISPIEL}

Fall 1: d-Transposition der großen Arterien (d-TGA) Während der Schwangerschaft waren beim Fetus die Diagnose einer d-Transposition der großen Arterien (d-TGA) gestellt und die Entbindung an einem Perinatalzentrum geplant worden. Bei der Spontangeburt war neben dem neonatologischen Team geplant auch eine Kinderkardiologin anwesend. Während eine Neonatologin einen Nabelvenenkatheter legte und mit der Volumensubstitution begann, kümmerte sich ihr Kollege um die Intubation und die maschinelle Beatmung. Nach diesen Maßnahmen stieg die präduktal gemessene pulsoxymetrische Sauerstoffsättigung $\left(\mathrm{S}_{\mathrm{p}} \mathrm{O}_{2}\right)$ auf $55 \%$, die postduktale auf $64 \%$. Eine Erhöhung des $\mathrm{F}_{\mathrm{i}} \mathrm{O}_{2}$ von 0,21 auf 0,4 führte nur zu einer geringen Verbesserung. Die Kinderkardiologin bestätigte echokardiografisch die Diagnose einer d-TGA. Der Ductus arteriosus war offen, das Foramen ovale aber hoch restriktiv, sodass das Team die Indikation zur Ballonatrioseptostomie (BAS) stellte.

Der Neonatologe verabreichte eine Analgosedierung über den inzwischen gelegten periphervenösen Zugang. Unter echokardiografischer Führung durch dessen Kollegin führte die Kinderkardiologin die BAS über die Nabelvene durch. Die präduktale $\mathrm{S}_{\mathrm{p}} \mathrm{O}_{2}$ stieg auf $76 \%$, die postduktale auf $84 \%$. Das Neugeborene wurde auf die neonatologische Intensivstation verlegt, wo es nach 6 Stunden extubiert werden konnte. Eine Dauerinfusion mit Prostaglandin $\mathrm{E}_{1}$ wurde mit $5 \mathrm{ng} / \mathrm{kgKG} / \mathrm{min}$ fortgeführt.

Der Nahrungsaufbau verlief unkompliziert. Nach der Korrektur-OP am 6. Lebenstag konnte das Kind am 12. postoperativen Tag nach Hause entlassen werden. 


\section{Ballonatrioseptostomie}

Rashkind und Miller beschrieben 1966 die Ballonatrioseptostomie (BAS) bei einem zyanotischen Kind mit d-Transposition der großen Arterien (d-TGA) als erste kinderkardiologische Katheterintervention [13]. Die BAS ist heute eine der häufigsten Katheterinterventionen beim Neugeborenen und wird meist echogestützt (ohne Fluoroskopie) bettseitig auf der Intensivstation durchgeführt, oder wie in Kasuistik 1 geschildert, bereits im neonatologischen Erstversorgungsraum neben dem Kreißsaal.

Die typische Indikation für die BAS ist eine restriktive Vorhofkommunikation bei Neugeborenen mit einer $d$ TGA oder Herzfehlern mit einer ähnlichen Hämodynamik. Die Diagnose wird echokardiografisch gestellt.

Die im Fallbeispiel 1 beschriebene Situation stellt einen idealen Verlauf dar: Die Diagnose war pränatal bekannt, und ein komplettes Team aus Geburtshilfe, Neonatologie und Kinderkardiologie stand geplant zur Geburt bereit. Ein solches Vorgehen erscheint sinnvoll, da mit den gegenwärtig verfügbaren Methoden pränatal keine sichere Vorhersage über den zu erwartenden Verlauf der postnatalen Anpassung im Allgemeinen sowie die Größe und Funktionalität des offenen Foramen ovale (PFO) im Besonderen getroffen werden kann [14].
Die Vermeidung einer tiefen Hypoxie bei Kindern mit restriktivem PFO rechtfertigt aus klinischer Sicht den erhöhten Organisations- und Personalaufwand. Allerdings ist auch eine nicht gerechtfertigte Zunahme der Behandlungsintensität denkbar, wenn die Indikation zur BAS vor Optimierung der konservativen Therapie gestellt wird. Diese besteht im Wesentlichen aus einer Dauerinfusion mit Prostaglandin $\mathrm{E}_{1}$ sowie einer Volumensubstitution und erlaubt in den meisten Fällen eine semielektive Planung der Intervention.

Beim Neugeborenen bietet sich die Nabelvene als idealer Zugangsweg an. Unter sonografischer Kontrolle wird der Katheter über den Ductus venosus zunächst in den rechten und dann über das PFO weiter in den linken Vorhof geschoben. Anschließend wird der Ballonkatheter gefüllt, mit einem kurzen Ruck über das Vorhofseptum in den rechten Vorhof gezogen und wieder entleert ( $\triangleright$ Video 1$)$. Das Interventionsergebnis wird echokardiografisch kontrolliert ( $\vee$ Video 2, $\vee$ Video 3 ). Meist kann ein unmittelbarer Anstieg der $\mathrm{S}_{\mathrm{p}} \mathrm{O}_{2}$ beobachtet werden.

Wenn bei älteren Kindern die Nabelvene nicht mehr sondierbar ist oder der Ductus venosus nicht passiert werden kann, wird der Eingriff über einen femoralvenösen Zugang durchgeführt.

\section{VIDEOS ONLINE!}

Sie finden die Videos unter www.thieme-connect. de/products bei Ihrer Neonatologie Scan.

\section{INFOBOX}

d-Transposition der großen Arterien (d-TGA)

- Kategorie:

- kritischer AHF

- Häufigkeit:

- zweithäufigster zyanotischer Herzfehler; 2,2\% aller AHF [1]

- Anatomie:

- Abgänge der großen Arterien sind vertauscht (Aorta aus RV, Pulmonalarterie aus LV) und stehen parallel zueinander (überkreuzen sich nicht)

- einfache d-TGA:

- eventuell Koronaranomalien

- keine weiteren Fehlbildungen

- komplexe d-TGA:

- Assoziation mit weiteren Fehlbildungen wie VSD, LVOTO, ISTA
- Pathophysiologie:

- einfache d-TGA: Pulmonal- vom Systemkreislauf getrennt (parallel geschaltet)

- Überleben nur mit Verbindungen zwischen den Kreisläufen (Shunts: PDA, PFO) möglich

- Therapie:

- präoperativ:

- Dauerinfusion mit Prostaglandin $\mathrm{E}_{1}$

- Optimierung Volumenstatus

- hoch normaler Hämatokrit

- ggf. BAS

- angestrebte $\mathrm{S}_{\mathrm{p}} \mathrm{O}_{2}$ um $80 \%$

- OP:

- anatomische Korrektur („arterieller Switch“) in den ersten beiden Lebenswochen

- Prognose:

- sehr gut bezüglich Lebenserwartung und -qualität 


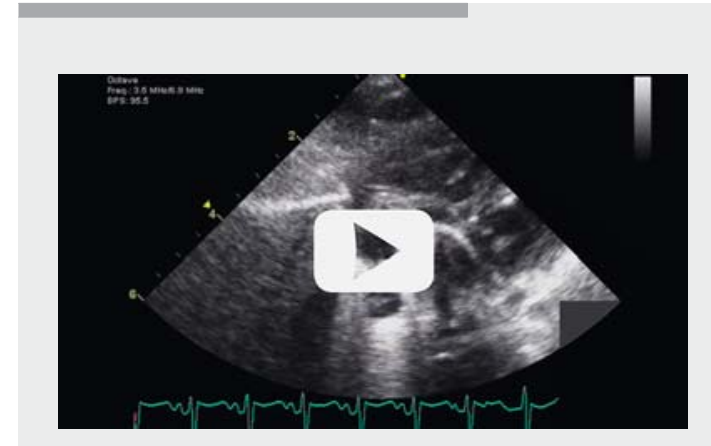

$\checkmark$ Video 1 Ballonatrioseptostomie nach Rashkind bei einem Neugeborenen mit d-Transposition der großen Gefäße (echokardiografische Darstellung von subkostal): Der mit physiologischer Kochsalzlösung gefüllte Ballon befindet sich im linken Vorhof. Von dort wird er mit einem kurzen Ruck über das Vorhofseptum in den rechten Vorhof gezogen.
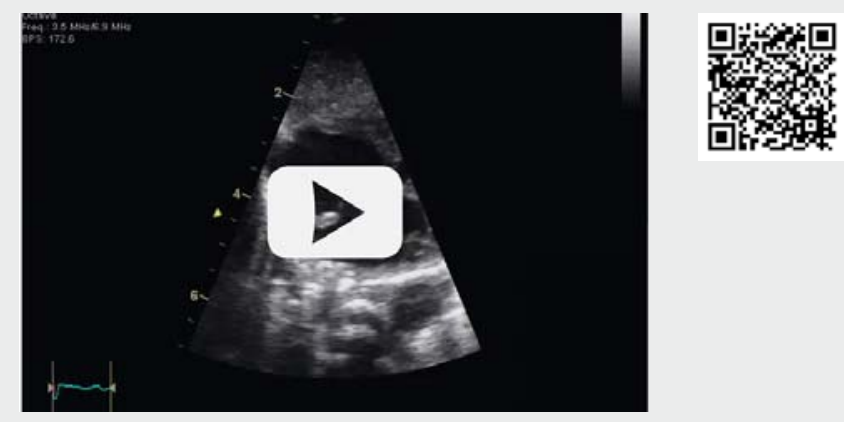

Video 2 Nach Ballonatrioseptostomie: große Vorfhofkommunikation.
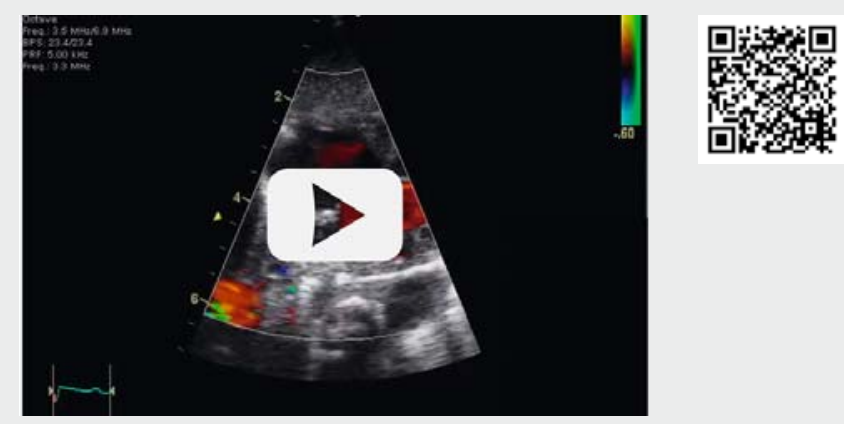

Video 3 Nach Ballonatrioseptostomie: große Vorfhofkommunikation (s. \ Video 2) mit farbdopplersonografisch kräftigem, nicht restriktiven Fluss.

\section{Cave}

Neugeborene mit kritischen angeborenen Herzfehlern sind postnatal von einer plötzlichen Verschlechterung ihres Allgemeinzustandes bis hin zum kardiogenen Schock, Multiorganversagen und Tod bedroht.

Cave

In den aktuellen Mutterschaftsrichtlinien ist lediglich die Darstellung des Vierkammerblicks gefordert; dieser ist bei d-TGA typischerweise unauffällig.

\section{Ballonvalvuloplastie}

\section{Pulmonalklappe}

Seit ihrer Einführung Anfang der 1980er-Jahre hat sich die Ballonvalvuloplastie der Pulmonalklappe, unabhängig vom Alter des Patienten, zur Methode der Wahl bei der Behandlung der isolierten Pulmonalklappenstenose entwickelt $[15,16]$. Beim Neugeborenen ist sie vor allem bei der kritischen Pulmonalstenose indiziert (s. Infobox).

Über einen femoralvenösen Zugang werden der rechte Vorhof und der rechte Ventrikel unter fluoroskopischer Kontrolle (Durchleuchtung) sondiert. Die Position der Pulmonalklappe und die Größe des Klappenrings lassen sich gut im seitlichen Strahlengang beurteilen ( Abb.2).

Über einen in der Peripherie des pulmonalarteriellen Gefäßbettes oder in der (über den Ductus arteriosus erreichbaren) Aorta descendens belassenen Führungsdraht wird ein Ballonkatheter vorgeschoben, sodass die Mitte des Ballons ungefähr auf Höhe des Klappenrings zu liegen kommt. Der Durchmesser des Ballons wird meist etwas größer (um ca. 20-30\%) als der des Klappenrings gewählt. Die verfügbaren Niederdruckballons passen durch Schleusen mit einem Durchmesser von 4 bzw. $5 \mathrm{Fr}$ ( $\triangleright$ Abb. 3). Ihr Arbeitsdruck liegt in einem Bereich um 3-4 atm. Während der Inflation des Ballons kann das Verstreichen einer Kerbe auf Höhe der Stenose fluoroskopisch beobachtet werden ( Video4).

Die kurz- und mittelfristigen Ergebnisse sind sehr gut [17-19]. Langfristig muss auch auf die Entwicklung einer Pulmonalinsuffizienz geachtet werden [20]. 

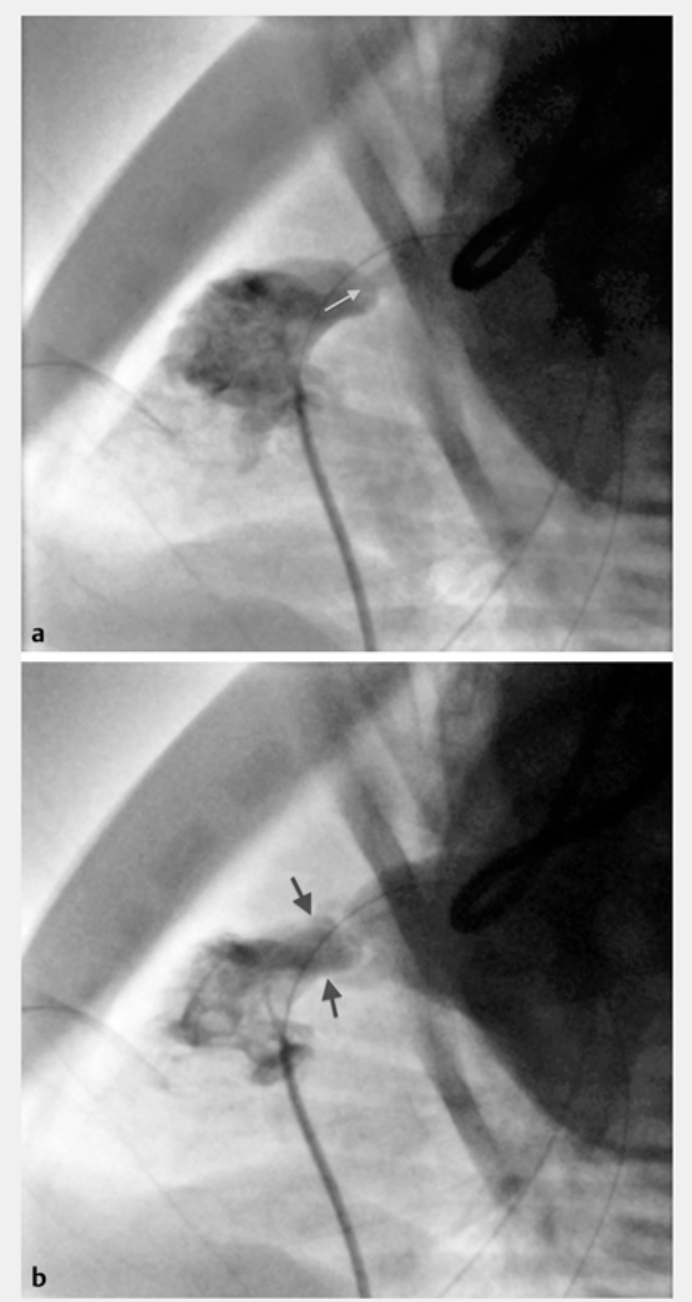

- Abb. 2 Kontrastmitteldarstellung im rechtsventrikulären Ausflusstrakt bei einem Neugeborenen mit kritischer valvulärer Pulmonalstenose (seitlicher Strahlengang). a Die Klappe bildet eine Kuppel mit einer minimalen Öffnung (Pfeil). b Wenn das Kontrastmittel die Pulmonalarterie erreicht hat, sind die Struktur der Klappe und die Größe ihres Rings (Pfeile) besser abgrenzbar.

\section{Merke}

Die meisten kritischen angeborenen Herzfehler können bereits mit echokardiografischen Grundkenntnissen nachgewiesen oder zumindest vermutet werden.

Wichtige Ausnahme: Abgrenzung einer totalen Lungenvenenfehlmündung mit Obstruktion von der persistierenden pulmonalen Hypertension des Neugeborenen (PPHN).

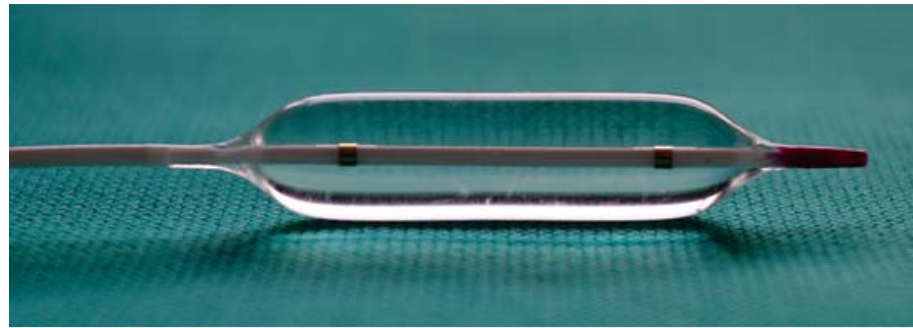

- Abb. 3 Niederdruckballon zur Dilatation von Klappenstenosen. Nominaler Druck 3,5 bar, maximal 4 bar. Radioopaque Marker (Gold) helfen bei der Positionierung.
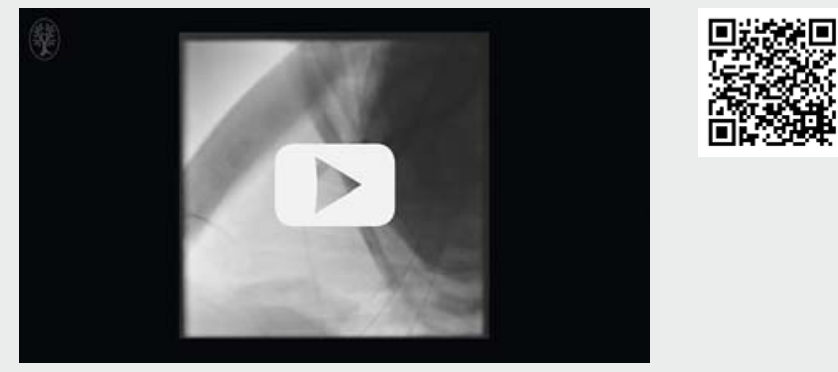

V Video 4 Ballonvalvuloplastie bei einem Neugeborenen mit kritischer Pulmonalstenose (seitlicher Strahlengang): Der Ballon bildet auf Höhe der Stenose eine Kerbe, die mit Erreichen des Arbeitsdruckes verstreicht.

INFOBOX

Kritische valvuläre Pulmonalstenose

- Kategorie:

- kritischer AHF

- Häufigkeit:

- bis $23 \%$ aller Patienten mit Pulmonalstenose [20]

- Anatomie:

- Stenose der Pulmonalklappe

- Pathophysiologie:

- Druckbelastung und Hypertrophie des RV

- in ausgeprägten Fällen ductusabhängige Lungenperfusion

- hochgradige Trikuspidalinsuffizienz

- Dilatation des rechten Vorhofes

- R/L-Shunt auf Vorhofebene

- systemische Untersättigung

- Differenzialdiagnose:

- assoziierte Fehlbildungen wie Hypoplasie der rechten Herzanteile

\section{- Therapie:}

- in Abhängigkeit vom Befund oder bei Verdacht: Prostaglandin $\mathrm{E}_{1}$

- Standardtherapie: Ballonvalvuloplastie der Pulmonalklappe semielektiv nach Diagnosestellung

\section{- Prognose:}

- sehr gut bezüglich Lebenserwartung und -qualität 


\section{Aortenklappe}

Die Ballonvalvuloplastie ist die an vielen Zentren bevorzugte Alternative zur Behandlung von Patienten mit kritischer Aortenklappenstenose. Dies gilt insbesondere für Neugeborene, deren linksventrikuläre Funktion bei Diagnosestellung bereits so stark beeinträchtigt ist, dass sie eine Herzinsuffizienz aufweisen oder sogar einen kardiogenen Schock erlitten haben; hier ist ein operatives Vorgehen mit Kommissurotomie und Rekonstruktion der Aortenklappe unter Einsatz der HerzLungen-Maschine mit einem deutlich erhöhten Risiko verbunden.

Die Entscheidung, ob Neugeborenen mit einer kritischen Aortenklappenstenose primär eine katheterinterventionelle oder eine operative Behandlung angeboten wird, hängt wesentlich von den Behandlungsergebnissen im jeweiligen Zentrum ab [21, 22].

Vor der Intervention ist eine detaillierte Echokardiografie erforderlich. Eine Kontraindikation zur Ballonvalvuloplastie kann sich aus einer assoziierten Hypoplasie der linken Herzanteile ergeben, die langfristig keine Etablierung einer biventrikulären Zirkulation erlaubt. Bei grenzwertig ausgeprägter Hypoplasie muss das therapeutische Vorgehen in enger Zusammenarbeit mit den Kinderherzchirurgen im Hinblick auf die Alternative einer univentrikulären Palliation erörtert werden.

\section{Merke}

Zur Planung der Behandlung von Neugeborenen mit kritischen angeborenen Herzfehlern ist eine umfassende, detaillierte Echokardiografie erforderlich.

\section{HINTERGRUNDWISSEN}

\section{Anmerkung}

Nach den aktuellen Leitlinien der Deutschen Gesellschaft für Pädiatrische Kardiologie bedarf ein Herzgeräusch beim Neugeborenen einer sofortigen Abklärung. Auch wenn dies im Text nicht explizit erwähnt ist, dürften damit v. a. Geräusche gemeint sein, die sich anhand des Untersuchungsbefundes und des - kurzfristig kontrollierten - klinischen Verlaufs nicht eindeutig als funktionell einordnen lassen. Die Planung der Abklärung eines bei Entlassung aus der Geburtsklinik persistierenden Herzgeräusches zur U3 kann für ein Neugeborenes mit kAHF allerdings deutlich zu spät und mit schwerwiegenden Folgen verbunden sein (s. klinisches Beispiel in > Abb.4).

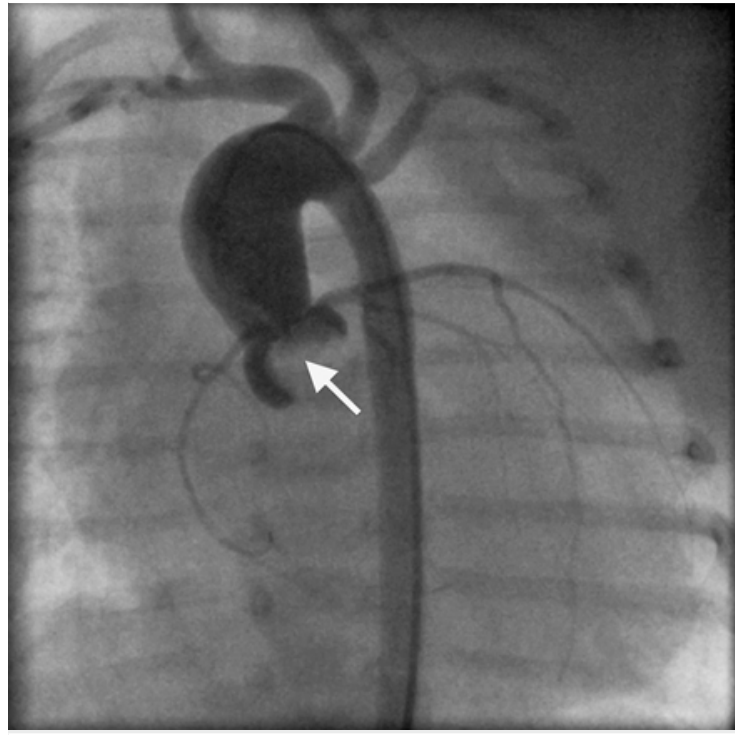

- Abb. 4 Aortografie (p. - a. Strahlengang) bei einem jungen Säugling mit kritischer Aortenklappenstenose. Die Aortenklappe bildet eine Kuppel mit einer winzigen zentralen Öffnung (Pfeil), über die (kontrastmittelfreies) Blut aus dem linken Ventrikel in die Aorta kommt. Nebenbefundlich ist eine ausgeprägte Kardiomegalie zu erkennen. Die Ventrikelgröße lässt sich auch aus den gleichzeitig mit angefärbten Koronarien abschätzen. Die Schwangerschaft kam nach assistierter Fertilisierung zustande. Die Feindiagnostik hatte einen unauffälligen Befund ergeben. Bei der U3 war ein Herzgeräusch festgestellt worden. Die Abklärung erfolgte im Alter von 7 Wochen. Nach sofortiger Einweisung ins Herzzentrum erfolgte die Ballonvalvuloplastie kurz nach der stationären Aufnahme. Klinisch hatte das Kind eine schwere Herzinsuffizienz. Der Eingriff führte zu einer klinischen Stabilisierung mit Normalisierung der Herzfunktion des inzwischen 4 Jahre alten Kindes.

Für den Eingriff kommen die gleichen Ballonkatheter wie zur Behandlung der Pulmonalklappe zum Einsatz. Der Durchmesser des Ballons wird allerdings etwas kleiner als der des Klappenringes (ca. 90\%) gewählt. Beim retrograden Zugang wird über einen femoralarteriellen Zugang die Aorta ascendens sondiert. Eine Angiografie zeigt die Morphologie der Aortenklappe, die meist eine Kuppel bildet und nur eine kleine Öffnung aufweist ( $\triangleright$ Abb.4). Diese wird mit einem feinen Führungsdraht sondiert, über den ein Katheter in den linken Ventrikel platziert werden kann. Nach Wechsel auf den Ballonkatheter kann die Valvuloplastie durchgeführt werden. 
Ein Vorteil des retrograden Zuganges liegt in der raschen Durchführbarkeit. Wesentlicher Nachteil ist die Notwendigkeit der Punktion der Femoralarterie, die mit dem Risiko einer postinterventionellen Perfusionsstörung verbunden ist.

Alternativ ist ein antegrades Vorgehen über einen femoralvenösen Zugang möglich. Die Sondierung des linken Ventrikels ist bei offenem Foramen ovale in der Regel unproblematisch. Zur Passage der Aortenklappe mit dem Draht und v. a. dem Ballonkatheter ist jedoch in Abhängigkeit von der Geometrie des linken Ventrikels - eine Richtungsänderung von fast $360^{\circ}$ nötig. Während der dafür erforderlichen Manipulationen besteht ein erhöhtes Risiko für eine zusätzliche hämodynamische Beeinträchtigung, z. B. durch Herzrhythmusstörungen oder Verschlechterung einer Mitralinsuffizienz. Grundsätzlich sind in geübten Händen beide Zugangswege möglich und führen zu vergleichbaren Ergebnissen [23].

\section{INFOBOX}

\section{Kritische valvuläre Aortenstenose}

- Kategorie:

- kritischer AHF

- Häufigkeit:

- ca. $10 \%$ aller Patienten mit Aortenstenose

- Anatomie:

- Stenose der Aortenklappe

- Pathophysiologie:

- eingeschränkte, manchmal PDA-abhängige Systemperfusion

- Druckbelastung des LV bis zum LV-Versagen

- beeinträchtigte Koronarperfusion durch reduzierten Systemdruck bei gleichzeitig erhöhtem LVEDP, MI

- postkapilläre PHT möglich

- Differenzialdiagnose:

- assoziierte Fehlbildungen: Hypoplasie linker Herzanteile (Aortenklappenring, LV, MK, LVOTO, ISTA)

- Therapie:

- Ballonvalvuloplastie oder operativ (Kommissurotomie/Aortenklappenrekonstruktion)

\section{- Prognose:}

- abhängig vom Ausmaß der vor Behandlungsbeginn eingetretenen Schädigung

- nach Normalisierung der LV-Funktion sehr gut bezüglich Lebenserwartung

- weitere Eingriffe mit hoher Wahrscheinlichkeit erforderlich
Ziel der Behandlung ist eine möglichst weitgehende Reduktion der Stenose ohne Ausbildung einer höhergradigen Insuffizienz, sodass sich die Herzfunktion erholen kann und - gegebenenfalls mit moderaten Restbefunden - längerfristig stabil bleibt. Inwieweit dies erreicht werden kann, hängt wesentlich von den oben beschriebenen anatomischen und funktionellen präinterventionellen Befunden ab.

Mittel- und langfristig sind mit sehr hoher Wahrscheinlichkeit weitere Eingriffe erforderlich. Die Vergleichbarkeit der Literatur zu dieser Frage ist u. a. durch Unterschiede in der Patientenauswahl, der Indikationsstellung und der Behandlungstechnik erschwert. In einer in Deutschland, Österreich und der Schweiz durchgeführten multizentrischen retrospektiven Studie lag der Anteil von Patienten, die nach neonataler Ballonvalvuloplastie der Aortenklappe eine operative Behandlung benötigten, nach 5 und 10 Jahren bei $30 \%$ bzw. $50 \%$ [24].

Merke

Hochgradige Stenosen von Pulmonal- und Aortenklappe können pränatal bei ausschließlicher Darstellung des Vierkammerblickes zumindest an indirekten Zeichen (z. B. Ventrikelhypertrophie, AV-Klappeninsuffizienz) vermutet werden.

Merke

Ein Herzgeräusch beim Neugeborenen bedarf einer sofortigen Abklärung (s.a. Infobox „Hintergrundwissen“).

\section{Radiofrequenzperforation der Pulmonalklappe}

Die Pulmonalatresie mit intaktem Ventrikelseptum (PA-IVS) zählt zu den kritischen angeborenen Herzfehlern mit ductusabhängigem Lungenkreislauf. Das anatomische Spektrum der Erkrankung ist ausgesprochen vielfältig (s. Infobox).

Bei ausgewählten Neugeborenen mit isolierter membranöser Atresie hat sich die Radiofrequenzperforation mit nachfolgender Ballonvalvuloplastie als Alternative zum chirurgischen Vorgehen etabliert. Je geringer die Hypoplasie des rechten Ventrikels und der Trikuspidalklappe ausgeprägt sind, desto höher ist die Wahrscheinlichkeit, langfristig eine biventrikuläre Zirkulation etablieren zu können. Weitere Voraussetzungen sind u.a. gut entwickelte zentrale Pulmonalarterien und das Fehlen einer Koronaranomalie, bei der die Reduktion des rechtsventrikulären Druckes die Koronarperfusion gefährdet (s. Infobox). Über die Indikation zur Intervention kann daher erst nach einer detaillierten Echokardiografie entschieden werden. 


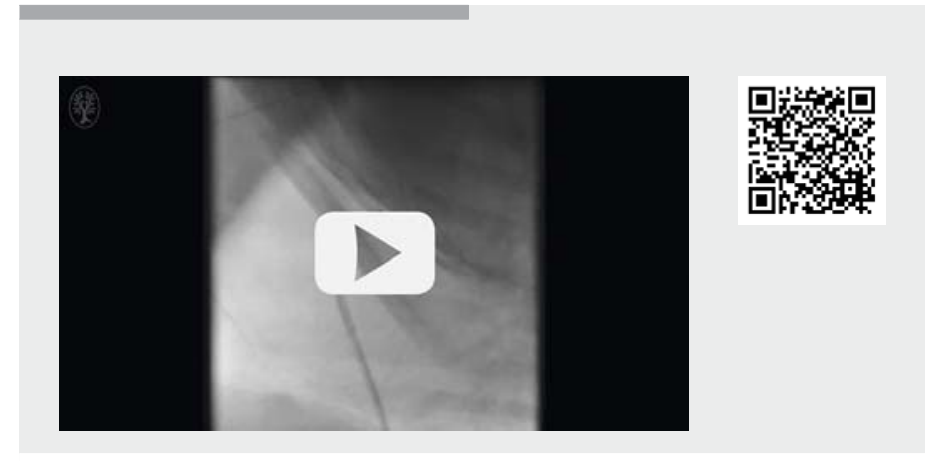

$\checkmark$ Video 5 Radiofrequenzperforation der Pulmonalklappe bei einem Neugeborenen mit Pulmonalatresie mit intaktem Ventrikelseptum (seitlicher Strahlengang): Ein endoffener Katheter liegt unmittelbar vor der Klappe. Unter Abgabe von mittels Radiofrequenz an der Spitze des Drahtes erzeugter Wärme wird die Klappe perforiert, sodass der Draht vorgeschoben werden kann.

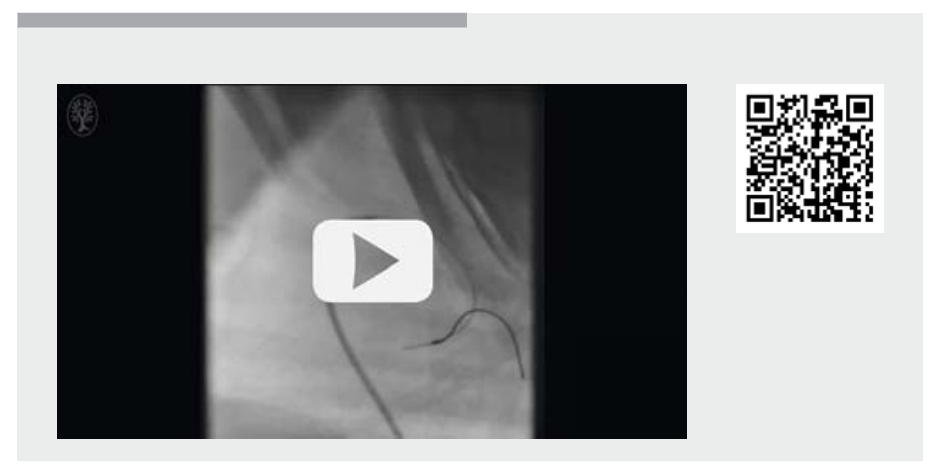

$\checkmark$ Video 6 Angiografie nach Radiofrequenz-Perforation der atretischen Pulmonalklappe mit Anfärbung der Pulmonalarterie und des rechten Ventrikels. Über eine noch bestehende Trikuspidalinsuffizienz fließt auch Kontrastmittel in den rechten Vorhof zurück.

Über einen femoralvenösen Zugang wird, ähnlich dem Vorgehen bei kritischer Pulmonalstenose, der rechte Ventrikel sondiert. Allerdings können ein kleines rechtsventrikuläres Volumen und eine ausgeprägte Hypertrophie die Sondierung erschweren. Größe, Struktur und Funktion des rechten Ventrikels und der Trikuspidalklappe, v. a. aber des rechtsventrikulären Ausflusstraktes, werden angiografisch beurteilt ( $\bullet$ Abb. 5).

Über einen endoffenen Katheter wird ein Radiofrequenzdraht idealerweise in der Mitte der atretischen Klappe platziert. Nach Erreichen der Zielposition wird er unter gleichzeitiger Energieabgabe (5-10W für ca. 2 s) über die Klappe bewegt ( $\triangleright$ Video 5 ). Ein besonderes Risiko des Eingriffes stellt die Perforation des rechtsventrikulären Ausflusstraktes mit Ausbildung eines Hämatoperikards bis hin zur Perikardtamponade oder eines Hämatothorax dar. Eine genaue Kontrolle der Draht- und Katheterposition ist daher unverzichtbar. Ein Ultraschallgerät sollte bereitstehen, um einen

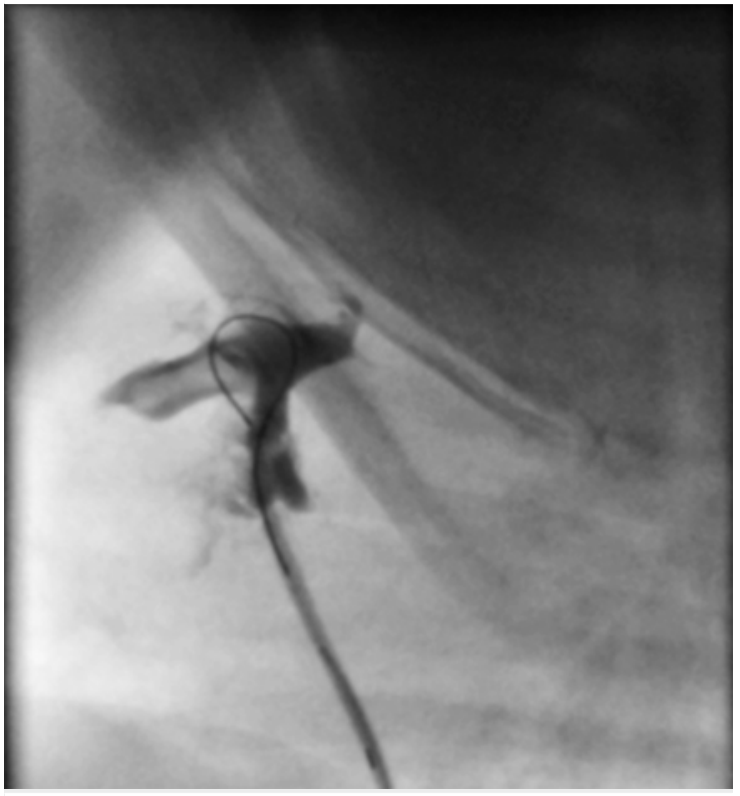

- Abb. 5 Kontrastmitteldarstellung des rechtsventrikulären Ausflusstraktes bei einem Neugeborenen mit Pulmonalatresie und intaktem Ventrikelseptum (seitlicher Strahlengang): Das Kontrastmittel verlässt den Ventrikel nicht: Die Pulmonalklappe ist atretisch.

Erguss ausschließen und ggf. eine Punktion oder Drainage unterstützen zu können.

Nach erfolgreicher Perforation der Klappe erfolgt eine sequenzielle Ballondilatation der Pulmonalklappe. Anschließend wird das Interventionsergebnis angiografisch kontrolliert ( $\vee$ Video 6 ).

Meist muss postinterventionell der Ductus arteriosus (PDA) weiter offen gehalten werden, um im Verlauf zu beurteilen, ob sich ein ausreichender antegrader Fluss über den rechtsventrikulären Ausflusstrakt entwickelt. Dies hängt nicht nur von dessen Größe und dem Erfolg der Klappeneröffnung ab, sondern auch vom Volumen, der Hypertrophie und der Compliance des rechten Ventrikels, außerdem von Größe und Funktion der Trikuspidalklappe. Die Entwicklung dieser Prozesse kann durchaus mehrere Wochen oder auch länger dauern, sodass, in Abhängigkeit von der Befundentwicklung, eine erneute Ballonvalvuloplastie und oder eine Stentimplantation im PDA erforderlich werden können. Ob die Versorgung des PDA mit einem Stent gleich beim ersten Eingriff erfolgen sollte, ist noch umstritten [25, 26]. 
INFOBOX

Pulmonalatresie mit intaktem Ventrikelseptum

- Kategorie:

- kritischer AHF

- Häufigkeit:

- 3-4 von 100000 Lebendgeborenen [1,27]

- Anatomie: breites Spektrum der Ausprägung, u.a. sind möglich:

- rein membranöse versus zusätzliche langstreckige infundibuläre Atresie

- unterschiedlich ausgeprägte Hypoplasie des RV

- Verbindungen (Sinusoide, Fisteln) zwischen RV und Koronarien

- Hypoplasie, Stenose und/oder Insuffizienz der Trikuspidalklappe

- Pathophysiologie:

- immer: PDA-abhängige Lungenperfusion, meist suprasystemischer Druck im rechten Ventrikel, R/L-Shunt auf Vorhofebene, Zyanose

- möglich: beeinträchtigte Koronarperfusion

- Therapie, Optionen stark abhängig von anatomischer Ausprägung:

1. bei isolierter membranöser Pulmonalatresie und gut entwickelten rechten Herzanteilen biventrikuläre Korrektur möglich:
- katheterinterventionell: Radiofrequenzperforation der Pulmonalklappe und sequenzielle Ballondilatation, langfristig ASD-Verschluss, Pulmonalklappenersatz

- operativ: Anlage eines modifizierten BlalockTaussig-Shunts, Eröffnung/Erweiterung des rechtsventrikulären Ausflusstraktes, langfristig: ASD-Verschluss, Pulmonalklappenersatz

2. bei hypoplastischen rechten Herzanteilen: univentrikuläre Palliation

3. bei Zwischenformen: ggf. Versuch einer Rekrutierung des RV für eine biventrikuläre Zirkulation und bei Bedarf späterer Wechsel auf Einkammer-oder 1,5-Kammer-Zirkulation

- Prognose: variabel:

- sehr gut bei isolierter PA-IVS mit biventrikulärer Funktion

- in manchen Fällen entscheidet sich erst nach längerer Zeit, ob eine biventrikuläre Zirkulation möglich ist

\section{Stentimplantation - Ductus} arteriosus

\section{Ductusabhängige Lungenperfusion}

Als chirurgisches Standardverfahren zur Sicherung der pulmonalen Perfusion bei Vitien mit ductusabhängiger Lungendurchblutung hat sich der modifizierte BlalockTaussig-Shunt etabliert und über viele Jahrzehnte bewährt. Dabei wird eine Goretex-Prothese zwischen dem arteriellen und dem pulmonalen Gefäßsystem implantiert: meist zwischen Truncus brachiocephalicus und dem ipsilateralen Ast der Pulmonalarterie.

Mit der Verfügbarkeit von kleinen, flexiblen Stents, die meist für die Behandlung von Koronargefäßen entwickelt wurden, besteht die Alternative, den Ductus arteriosus durch Implantation eines oder mehrerer Stents bis zum später erforderlichen operativen Eingriff offen zu halten.

Die zugrundeliegende Anatomie wird echokardiografisch untersucht und entscheidet über den optimalen Gefäßzugang: Bei Pulmonalatresie mit Ventrikelseptumdefekt kann eine antegrade Sondierung über einen venösen Zugang, die rechten Herzanteile, den Ventrikelseptumdefekt und die Aorta sinnvoll sein. Ansons- ten wird der Ductus arteriosus retrograd über einen arteriellen Zugang erreicht. Nach angiografischer Darstellung und Platzierung eines Führungsdrahtes in einem der Pulmonalarterienäste wird der auf einem Ballonkatheter vormontierte Stent in den Ductus geschoben. Die Implantation erfolgt durch Inflation des Ballons, der anschließend vorsichtig entfernt wird. Die korrekte Stentposition wird angiografisch und ggf. auch echokardiografisch dokumentiert ( Abb.6).

Die Entscheidung darüber, ob ein katheterinterventionelles oder ein chirurgisches Vorgehen gewählt wird, sollte im Behandlungsteam gemeinsam mit Vertretern beider Disziplinen getroffen werden. Dabei müssen die zugrunde liegende Anatomie, die Erfahrungen und Behandlungsergebnisse mit der jeweiligen Methode in der Institution und weitere Faktoren wie Alter und Gewicht des Patienten sowie ggf. assoziierte Erkrankungen Berücksichtigung finden.

Inzwischen konnte auch in einer größeren Studie belegt werden, dass die Stentimplantation in den Ductus arteriosus eine sinnvolle Alternative zur chirurgischen Anlage eines modifizierten Blalock-Taussig-Shunts sein kann [28]. 

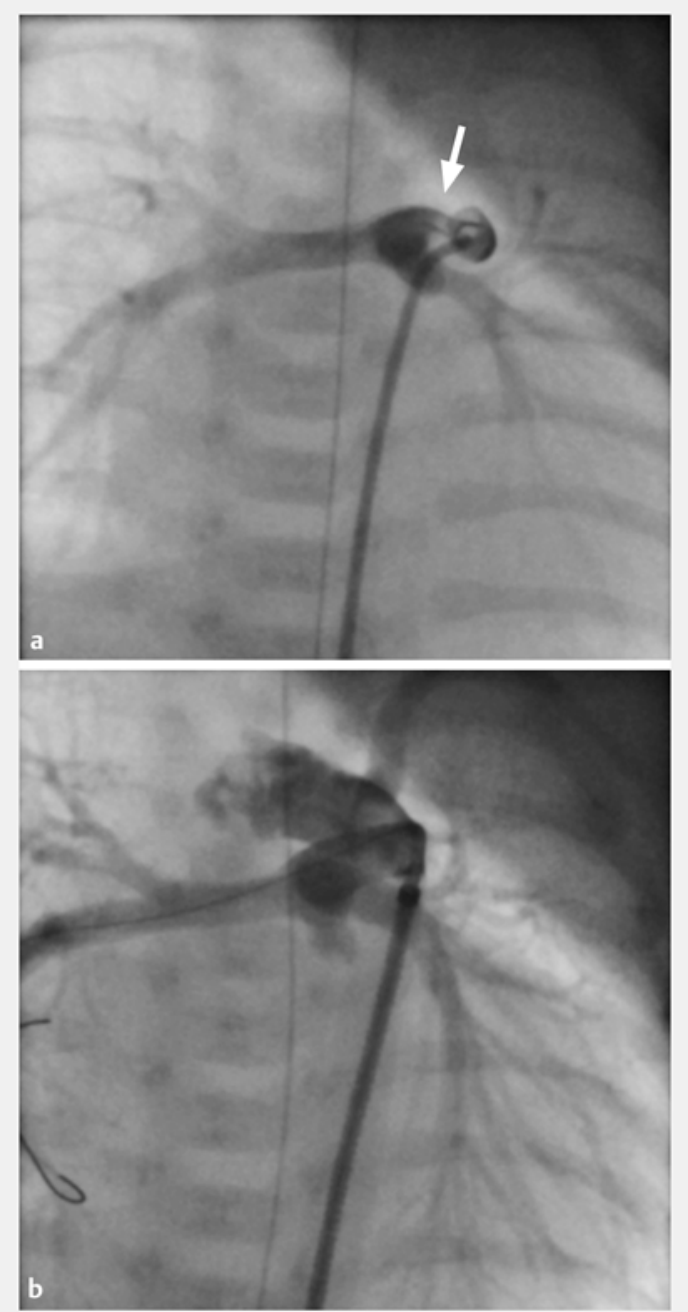

- Abb. 6 Stentimplantation als Alternative zu einem chirurgisch angelegten Shunt bei einem Neugeborenen mit hypoplastischem Rechtsherzsyndrom (p. - a. Strahlengang). a Der PDA weist unmittelbar vor der Mündung in die Pulmonalarterie eine Enge auf (Pfeil). b Regelrechte Position und Funktion des Stents im PDA.

\section{Hybridpalliation bei hypoplastischem Linksherzsyndrom - „Gießen-Prozedur“}

Trotz erheblicher Fortschritte ist die Behandlung von Patienten mit hypoplastischem Linksherzsyndrom mit einer bedeutsamen Mortalität und vor allem einer ausgeprägten Morbidität verbunden (s. Infobox).

Als Alternative zum ersten operativen Schritt, der univentrikulären Palliation nach Norwood, wurde ein Hybridverfahren entwickelt: Neben chirurgischer Anlage einer Bändelung beider Pulmonalarterienäste erfolgt eine Stentimplantation in den Ductus arteriosus. In Abhängigkeit von der zugrunde liegenden Anatomie ist zusätzlich eine Vergrößerung der Vorhofkommunikation und/oder eine Stentimplantation im Aortenisthmus erforderlich [29].
Als Hauptvorteil dieses Vorgehens wird die Vermeidung einer großen Herz-Lungen-Maschinen-Operation in der frühen Neonatalzeit angesehen; die erforderliche Rekonstruktion des Aortenbogens erfolgt erst nach einigen Monaten gemeinsam mit der oberen kavopulmonalen Anastomose [30].

Ein Nachteil des Verfahrens besteht in der aus der bilateralen Bändelung resultierenden Beeinträchtigung des Wachstums der Pulmonalarterien, deren adäquate Größe und Funktion wichtige Voraussetzung für die spätere univentrikuläre Palliation sind [31, 32]. In vielen Zentren wird die Hybridpalliation daher nur bei Patienten eingesetzt, bei denen das Risiko für eine klassische Norwood-1-Operation als zu hoch eingeschätzt wird [33, 34].

\section{INFOBOX}

Hypoplastisches Linksherzsyndrom

- Kategorie:

- kritischer AHF

- Häufigkeit:

- 15 von 100000 Lebendgeborene [1]

- Anatomie:

- Hypoplasie der linken Herzanteile (MK, LV, AK, Aorta ascendens, Aortenbogen)

- ISTA

- Sonderfall: restriktives/verschlossenes Vorhofseptum (s. Text)

\section{- Pathophysiologie:}

- PDA-abhängige Systemperfusion

- RV als Systemventrikel

- retrograde Perfusion der Aorta ascendens

- kritische Balance zwischen systemischer und pulmonaler Perfusion: bei sinkendem pulmonalem Widerstand pulmonale Überflutung, schwere Herzinsuffizienz, Koronarischämie

- Therapie, Optionen:

1. univentrikuläre Palliation nach Norwood in 3 operativen Schritten zur vollständigen Kreislauftrennung

2. Hybridpalliation (s. Text)

3. Verzicht auf lebensverlängernde Maßnahmen, „comfort care“

4. Herztransplantation (aufgrund des Mangels an Spenderorganen nicht realistisch)

\section{- Prognose:}

- bei 1) Erreichen des Erwachsenenalters in ca. 70\% der Fälle [35], an einzelnen Zentren z. T. höher

- bei restriktivem/verschlossenem Vorhofseptum deutlich schlechter

- erhebliche Morbidität 


\section{Merke}

Neugeborene mit hypoplastischem Linksherzsyndrom und hochgradig restriktivem oder verschlossenem Vorhofseptum sind unmittelbar nach Geburt akut bedroht.

\section{Dekompression des linken Vorhofes bei Patienten mit hypoplastischem Linksherzsyndrom mit restriktivem/ intaktem Vorhofseptum}

Unbehandelt versterben Neugeborene mit hypoplastischem Linksherzsyndrom meist in den ersten Lebenstagen. Wenn zusätzlich das Vorhofseptum hochgradig restriktiv oder sogar verschlossen ist, sind die Kinder bereits unmittelbar nach Geburt akut gefährdet: Das Blut staut sich vom linken Vorhof in die Lunge, deren Perfusion hochgradig eingeschränkt ist, was zu einer akuten refraktären Hypoxie führt, die nur durch Schaffung einer ausreichenden Vorhofkommunikation behoben werden kann.

Wenn die Diagnose pränatal bekannt ist, kann nach ausführlicher Beratung die Entbindung an einem Zentrum in Kooperation von Geburtshilfe, Anästhesie, Neonatologie, Kinderkardiologie und -chirurgie geplant werden. Nach Geburt, Erstversorgung und Bestätigung der Diagnose muss möglichst schnell eine ausreichend große Vorhofkommunikation geschaffen werden, um eine Stabilisierung zu ermöglichen.
Eine chirurgische Septektomie ist mit sehr hohem Risiko verbunden. Daher kommen bevorzugt katherinterventionelle Techniken zum Einsatz. Die Perforation des Vorhofseptums ist mit einer transseptalen Punktionsnadel oder einem RF-Draht möglich. Zur Vergrößerung des Defektes kommen Ballonkatheter in Betracht. Mit Implantation eines Stents lässt sich die Öffnung auf einen definierten Durchmesser anhaltend vergrößern [36,37] ( Abb. 7). Oft ist die Prozedur durch die hämodynamische Instabilität und die geringe Körpergröße der Patienten sowie durch die besonderen anatomischen Verhältnisse (verdicktes Vorhofseptum, kleiner linker Vorhof) erschwert [22].

Auch nach erfolgreicher Intervention bleibt die Prognose dieser Patienten schlechter, da die Anomalie des Vorhofseptums häufig bereits intrauterin teilweise irreversible Umbauvorgänge im Bereich der Lungengefäße verursacht hat, die langfristig die Funktion einer univentrikulären Palliation beeinträchtigen können [38]. Die bisherigen Ergebnisse von Versuchen, diese Prozesse bereits beim Fetus mit einer transuterinen Stentimplantation in das Vorhofseptum aufzuhalten, rechtfertigen noch keine Anwendung in der klinischen Routine $[39,40]$.

In Anbetracht der Schwere der Erkrankung kann es gegenwärtig als gerechtfertigt erscheinen, mit Familien, die sich gegen eine Unterbrechung der Schwangerschaft entschieden haben, aber auch zurückhaltend gegenüber einer aggressiven Maximaltherapie sind, eine begleitende Pflege unter Verzicht auf lebensverlängernde Maßnahmen nach der Geburt („compassionate care“) zu diskutieren.
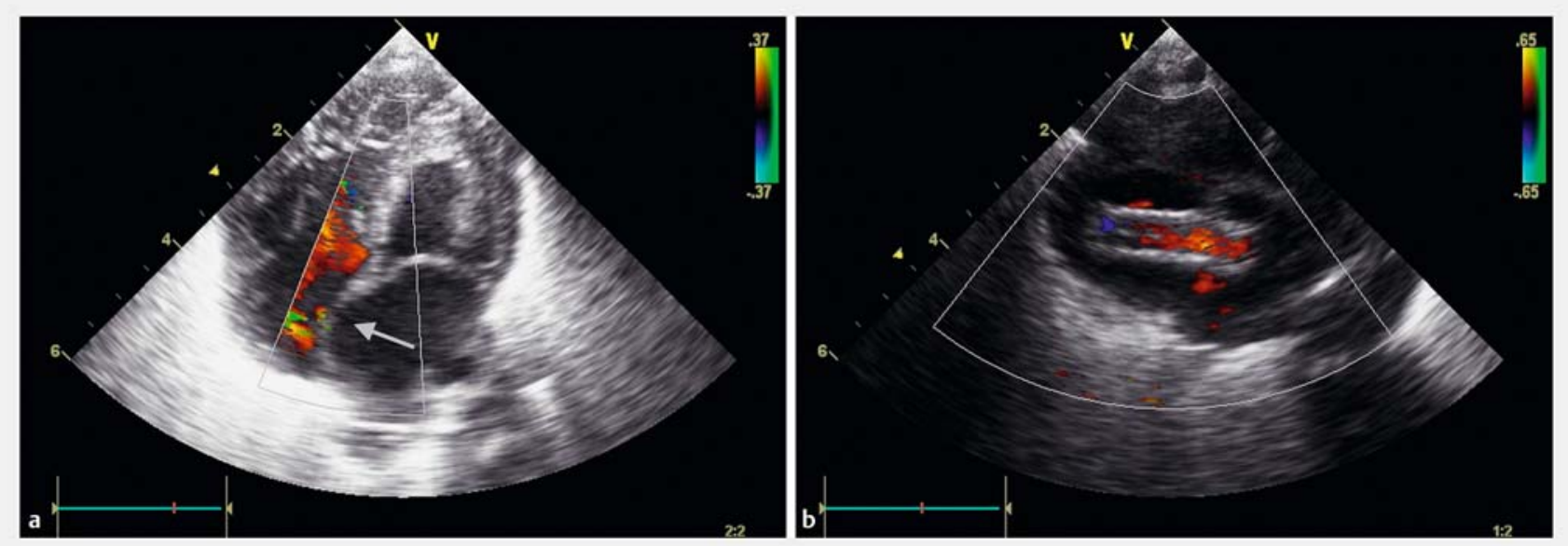

- Abb.7 Frühgeborenes mit hypoplastischem Linksherzsyndrom und pränatal bekanntem hoch restriktivem Foramen ovale. Die Entbindung erfolgte geplant im Herzzentrum. a Apikaler Vierkammerblick: Der linke Ventrikel ist hypoplastisch, der rechte spitzenbildend. Während die Trikuspidalklappe geöffnet ist, bleibt die Mitralklappe geschlossen. Im Bereich des Vorhofseptums ist farbdopplersongrafisch eine winzige Öffnung zu erahnen (Pfeil). b Nach Erstversorgung, Bestätigung der Diagnose und sofortiger Verlegung ins Herzkatheterlabor wurde nach transseptaler Punktion und Ballondilatation ein Stent in das Vorhofseptum implantiert. Die Echokardiografie zeigt eine regelrechte Stentposition mit farbdopplersongrafisch kräftigem, nicht restriktiven Fluss. 


\section{Weitere Katheterinterventionen}

\section{Aortenisthmusstenose}

Die Aortenisthmusstenose (ISTA) kann sowohl isoliert als auch in Kombination mit anderen Herzfehlern auftreten. Auch nach genauer pränataler Diagnosestellung ist postnatal eine Wiederholung der Echokardiografie zur Planung der Behandlung erforderlich. Die Standardtherapie der kritischen Aortenisthmusstenose ist die Operation.

Die in den U-Untersuchungen geforderte, wiederholte vergleichende Pulspalpation an oberen und unteren Extremitäten leistet einen wichtigen Beitrag zur Diagnostik der Aortenisthmusstenose.

Bei Neugeborenen, die aufgrund einer späten Diagnose bereits ein Herz-Kreislauf-Versagen oder einen kardiogenen Schock mit Multiorganversagen entwickelt haben, kann eine Ballondilatation des Aortenisthmus aber zur präoperativen Stabilisierung des Kindes beitragen. Bei hohem OP-Risiko ist auch eine Stentimplantation im Bereich des Aortenisthmus möglich, aber mit der Notwendigkeit wachstumsbedingter Folgeeingriffe verbunden.

\section{Verschluss des Ductus arteriosus}

Der katheterinterventionelle Verschluss ist die Methode der Wahl beim behandlungsbedürftigen Ductus arteriosus jenseits der Früh- und Neugeborenenperiode.

Grundsätzlich ist ein katheterinterventioneller Verschluss auch bei Neu- und sehr kleinen Frühgeborenen möglich [41,42]. In Anbetracht der fortbestehenden Unklarheiten bezüglich des optimalen Vorgehens beim Ductus arteriosus des Frühgeborenen sollte die Indikation zum katheterinterventionellen Verschluss in dieser Altersgruppe vorerst nur in Einzelfällen nach sorgfältiger Abwägung des Risiko-Nutzen-Verhältnisses gestellt werden.

\section{Verschluss vaskulärer Anomalien}

In seltenen Fällen können gefäßreiche Tumoren bereits im Neugeborenenalter zu einer schweren Herzinsuffizienz führen. Eine katheterinterventionelle Embolisation der Gefäße ist mit verschiedenen Implantaten möglich ( $\triangleright$ Abb. 8). Auch bei arteriellen Gefäßen, die einen Lungensequester (z. B. beim Scimitar-Syndrom) versorgen, oder bei aortopulmonalen Kollateralen, die mit verschiedenen Herzfehlern auftreten, kann ein katheterinterventioneller Verschluss in der Neugeborenenzeit indiziert sein.

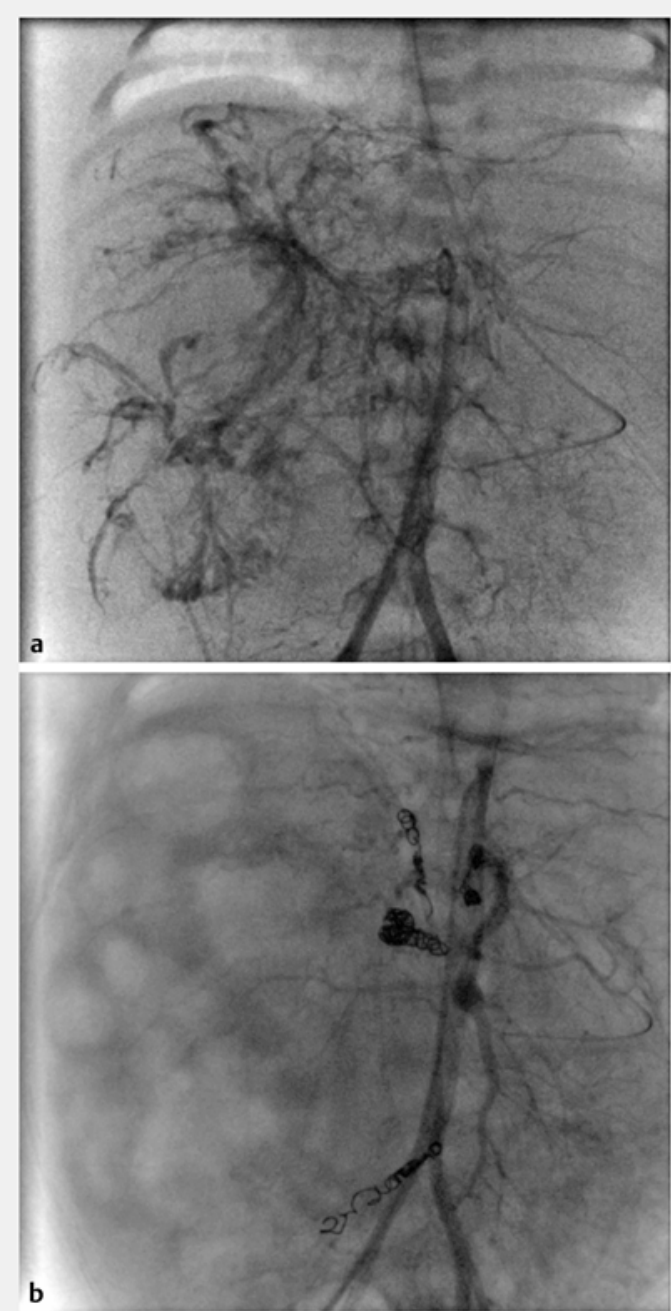

- Abb. 8 Neugeborenes mit riesigem Lebertumor (V.a. Hämangioendotheliom), Kasabach-Meritt-Syndrom, schwerer kardiorespiratorischer Insuffizienz und beginnendem Multiorganversagen. a Eine Angiografie in der Aorta abdominalis (p. - a. Strahlengang) lässt das Ausmaß der Gefäßversorgung des Tumors erkennen. b Nach Embolisation (insgesamt 28 Coils) von 4 der größeren Tumorgefäße deutliche Besserung. Postinterventionell kam es zu einer raschen Stabilisierung. Nach Rückverlegung in die Neonatologie konnte das Kind am 3. postinterventionellen Tag extubiert werden.

\section{Postoperative Katheterinterventionen}

In vielen Fällen können Katheterinterventionen dazu beitragen, das Ergebnis nach einer Herzoperation zu verbessern oder zu erhalten. So können Stenosen von Gefäßen oder chirurgisch angelegten Shunts mittels Ballondilatation und/oder Stentimplantation behandelt werden. 


\section{Merke}

Herzkatheterinterventionen können bei herzoperierten Kindern dazu beitragen, Re-Operationen zu vermeiden.

\section{Bergung von Fremdkörpern}

Nicht nur dislozierte Implantate, sondern z. B. auch abgerissene Teile von zentralvenösen Kathetern können katheterinterventionell geborgen werden.

\section{Rhythmologische Katheterinterventionen}

Über eine transvenöse Sonde kann der rechte Ventrikel bei symptomatischem AV-Block III temporär stimuliert werden. Typische rhythmologische Interventionen wie Radiofrequenz- oder Kryoablationen spielen im Neugeborenenalter keine Rolle.

\section{Herzkatheterinterventionen beim Frühgeborenen}

Herzkatheterinterventionen sind auch bei Kindern mit niedrigem, sehr niedrigem und sogar extrem niedrigem Geburtsgewicht möglich [5-7,41,42]. Allerdings ist das periinterventionelle Risiko bei Kindern in dieser Alters- und Gewichtsklasse erhöht. Die Indikation sollte daher im Einzelfall in enger Abstimmung zwischen dem neonatologischen, dem kinderkardiologischen und dem kardiochirurgischen Team gestellt werden.

\section{Merke}

Frühgeborene mit angeborenen Herzfehlern profitieren besonders von einer engen interdisziplinären Zusammenarbeit zwischen Neonatologie, Kinderkardiologie und -herzchirurgie.

\section{FALLBEISPIEL}

\section{Fall 2: Frühgeborenes mit Fallot-Tetralogie}

Während der Schwangerschaft war bei einem Zwilling eine Fallot-Tetralogie diagnostiziert und die Entbindung im Perinatalzentrum mit Kinderkardiologie/-herzchirurgie geplant worden. Nach vorzeitigem Blasensprung erfolgte die Geburt jedoch mit einem Gestationsalter von 29+2/7 SSW im heimatnahen Perinatalzentrum.

Beim ersten Zwilling (GG $1120 \mathrm{~g}$ ) wurde die Diagnose der Fallot-Tetralogie bestätigt. Nach der Erstversorgung mit Behandlung eines Atemnotsyndroms und nach Absinken des pulmonalen Widerstandes bestand eine pulmonale Überflutung mit hohen Sauerstoffsättigungen. Der Ductus arteriosus verschloss sich spontan. Das Kind war ohne Atemhilfe stabil, entwickelte aber in der 4. Lebenswoche eine zunehmende infundibuläre Pulmonalstenose mit Zyanose. Nach Verlegung ins Herzzentrum wurde - bei einem Gewicht von $1640 \mathrm{~g}$ - die Indikation zur Stentimplantation im Bereich des rechtsventrikulären Ausflusstraktes gestellt. Der Eingriff verlief ohne Komplikationen und führte zu einem Anstieg der Sauerstoffsättigung; das Kind konnte am 1. postinterventionellen Tag in die heimatnahe Betreuung zurückverlegt werden.

In der 9. Lebenswoche hatte sich bei einem Gewicht von $2310 \mathrm{~g}$ eine symptomatische muskuläre Enge unterhalb des Stents entwickelt, die durch Implantation eines zweiten Stents beseitigt werden konnte. Die Stents wurden im Alter von 7 Monaten nachdilatiert. Die Korrektur-OP - mit komplikationsloser Entfernung der Stents - erfolgte elektiv im Alter von 8 Monaten und bei einem Gewicht von $6100 \mathrm{~g}$.

\section{KERNAUSSAGEN}

- Herzkatheterinterventionen haben einen festen Stellenwert in der Behandlung von Neugeborenen mit kritischen angeborenen Herzfehlern.

- Die Ballonvalvuloplastie der Pulmonalklappe stellt eine eigenständige Behandlung dar.

- Häufig sind Herzkatheterinterventionen beim Neugeborenen Teil eines Behandlungskonzeptes, bei dem operative Techniken zentral eingesetzt werden.

- Mit Hilfe von Herzkatheterinterventionen kann häufig der präoperative Zustand stabilisiert oder das OP-Ergebnis erhalten bzw. verbessert werden.

- In Zentren mit entsprechender Erfahrung können auch Eingriffe bei Neu- und sogar bei Frühgeborenen sicher und mit geringem Risiko durchgeführt werden.

- Vor allem nach Verwendung arterieller Zugänge im Neugeborenenalter müssen postinterventionell Gefäßverschlüsse sonografisch ausgeschlossen werden. 


\section{Schlüsselwörter}

Neugeborenes, kritischer angeborener Herzfehler, Herzkatheterintervention

Interessenkonflikt

Erklärung zu finanziellen Interessen

Forschungsförderung erhalten: nein; Honorar/geldwerten Vorteil für Referententätigkeit erhalten: nein; Bezahlter Berater/interner Schulungsreferent/Gehaltsempfänger: nein; Patent/Geschäftsanteile/Aktien (Autor/Partner, Ehepartner, Kinder) an Firma: nein.

Erklärung zu nichtfinanziellen Interessen

Die Autoren geben an, dass kein Interessenkonflikt besteht.

\section{Autorinnen/Autoren}

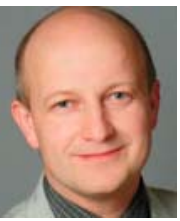

\section{Frank-Thomas Riede}

Dr. med., Jahrgang 1967. 1986-1993 Studium der Humanmedizin an der FAU Erlangen-Nürnberg und der Université de Rennes, Frankreich. Facharzt für Kinder- und Jugendmedizin 2002, Teilgebiete: Neonatologie 2003, Kinderkardiologie 2005, Weiterbildungen: Spezielle Pädiatrische Intensivmedizin 2007, Erwachsene mit angeborenen Herzerkrankungen 2014. Seit 2012 leitender Oberarzt an der Universitätsklinik für Kinderkardiologie am Herzzentrum Leipzig.

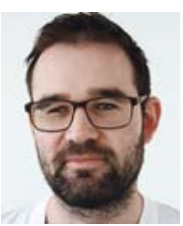

\section{Christian Paech}

Dr. med., Jahrgang 1982. 2002-2008 Studium der Humanmedizin an der Ernst-Moritz-Arndt Universität Greifswald und der Carl-Gustav-Carus Universität Dresden. Facharzt für Kinder- und Jugendmedizin 2014, Schwerpunkt Kinderkardiologie 2016, Zusatzbezeichnung Sportmedizin 2019. Seit 2016 Funktionsoberarzt am Herzzentrum Leipzig.

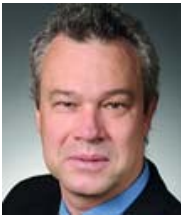

\section{Ingo Dähnert}

Prof. Dr. med., Jahrgang 1961. 1981 1987 Medizinstudium in Leipzig. Facharzt für Pädiatrie 1992, Kinderkardiologe 1994. Weiterbildungen in Pädiatrischer Intensivmedizin und der Behandlung Erwachsener mit angeborenen Herzerkrankungen. 1994-2000 Oberarzt im Deutschen Herzzentrum Berlin. 2000-2010 leitender Oberarzt der Universitätsklinik für Kinderkardiologie am Herzzentrum Leipzig, seit 2011 Klinikdirektor und W3-Professor.

\section{Korrespondenzadresse}

Dr. med. Frank-Thomas Riede

Universitätsklinik für Kinderkardiologie

Herzzentrum Leipzig

Strümpellstraße 39

04289 Leipzig

E-Mail: frank-thomas.riede@medizin.uni-leipzig.de

Wissenschaftlich verantwortlich gemäß Zertifizierungsbestimmungen

Wissenschaftlich verantwortlich gemäß Zertifizierungsbestimmungen für diesen Beitrag ist Dr. med. Frank-Thomas Riede, Leipzig

Literatur

[1] Lindinger A, Schwedler G, Hense HW. Prevalence of congenital heart defects in newborns in germany: results of the first registration year of the PAN Study (July 2006 to June 2007). Klin Pädiatr 2010; 222: 321 - 326

[2] Bergersen L, Gauvreau K, Foerster SR et al. Catheterization for congenital heart disease adjustment for risk method (CHARM). JACC Cardiovasc Intervent 2011; 4: 1037- 1046

[3] Mehta R, Lee K-J, Chaturvedi R et al. Complications of pediatric cardiac catheterization: A review in the current era. Cathet Cardiovasc Intervent 2008; 72: $278-285$

[4] Nykanen DG, Forbes T], Du W et al. CRISP: Catheterization RISk score for pediatrics: a report from the Congenital Cardiac Interventional Study Consortium (CCISC). Catheter Cardiovasc Interv 2016; 87: $302-309$

[5] Kobayashi D, Sallaam S, Aggarwal S et al. Catheterizationbased intervention in low birth weight infants less than $2.5 \mathrm{~kg}$ with acute and long-term outcome. Cathet Cardiovasc Intervent 2013; 82: $802-810$

[6] Kretschmar O, Dähnert I, Berger F et al. Interventionelle Behandlung angeborener Herzfehler bei einem Körpergewicht bis 2500 Gramm. Clin Res Cardiol 2000; 12: 1126 1132

[7] Sutton N, Lock JE, Geggel RL. Cardiac catheterization in infants weighing less than 1,500 grams. Cathet Cardiovasc Intervent 2006; 68: 948-956

[8] Brotschi B, Hug MI, Kretschmar O et al. Incidence and predictors of cardiac catheterisation-related arterial thrombosis in children. Heart 2015; 101: 948-953

[9] Kamyszek RW, Leraas H], Nag UP et al. Routine postprocedure ultrasound increases rate of detection of femoral arterial thrombosis in infants after cardiac catheterization. Catheter Cardiovasc Interv 2018; 93: 652-659

[10] Knirsch W, Kellenberger C, Dittrich S et al. Femoral arterial thrombosis after cardiac catheterization in infancy: impact of doppler ultrasound for diagnosis. Pediatr Cardiol 2012; 34: $530-535$

[11] Bontadelli J, Moeller A, Schmugge M et al. Enoxaparin therapy for arterial thrombosis in infants with congenital heart disease. Intensive Care Med 2007; 33: 1978- 1984

[12] Glatz AC, Keashen R, Chang J et al. Outcomes using a clinical practice pathway for the management of pulse loss follo- 
wing pediatric cardiac catheterization. Catheter Cardiovasc Interv 2015; 85: 111 - 117

[13] Rashkind WJ, Miller WW. Creation of an atrial septal defect without thoracotomy: a palliative approach to complete transposition of the great arteries. JAMA 1966; 196: 991 992

[14] Słodki M, Respondek-Liberska M, Pruetz JD et al. Fetal cardiology: changing the definition of critical heart disease in the newborn. J Perinatol 2016; 36: 575-580

[15] Kan JS, White RI, Mitchell SE et al. Percutaneous balloon valvuloplasty: a new method for treating congenital pulmonary-valve stenosis. N Engl J Med 1982; 307: 540-542

[16] Kreutzer]. Transcatheter intervention in the neonate with congenital heart disease. Clin Perinatol 2001; 28: 137 - 157

[17] Colli AM, Perry SB, Lock JE et al. Balloon dilation of critical valvar pulmonary stenosis in the first month of life. Cathet Cardiovasc Diagn 1995; 34: $23-28$

[18] Rao PS. Percutaneous balloon pulmonary valvuloplasty: State of the art. Cathet Cardiovasc Intervent 2007; 69: $747-763$

[19] Voet A, Rega F, de Bruaene AV et al. Long-term outcome after treatment of isolated pulmonary valve stenosis. International J Cardiol 2012; 156: 11 - 15

[20] Devanagondi R, Peck D, Sagi J et al. Long-term outcomes of balloon valvuloplasty for isolated pulmonary valve stenosis. Pediatr Cardiol 2017; 38: 247 - 254

[21] Franchi E, Cantinotti M, Assanta $\mathrm{N}$ et al. State of the art and prospective for percutaneous treatment for left ventricular outflow tract obstruction. Prog Pediatr Cardiol 2018; 51 : $55-61$

[22] Siddiqui ], Brizard CP, Galati JC et al. Surgical valvotomy and repair for neonatal and infant congenital aortic stenosis achieves better results than interventional catheterization. JAm Coll Cardiol 2013; 62: 2134-2140

[23] Mozumdar N, Burke E, Schweizer M et al. A comparison of anterograde versus retrograde approaches for neonatal balloon aortic valvuloplasty. Pediatr Cardiol 2018; 39: 450 458

[24] Ewert P, Bertram H, Breuer J et al. Balloon valvuloplasty in the treatment of congenital aortic valve stenosis - A retrospective multicenter survey of more than 1000 patients. International J Cardiol 2011; 149: 182 - 185

[25] Kutty S, Zahn EM. Interventional therapy for neonates with critical congenital heart disease. Cathet Cardiovasc Intervent 2008; 72: 663-674

[26] Alwi M, Choo K-K, Radzi NAM et al. Concomitant stenting of the patent ductus arteriosus and radiofrequency valvotomy in pulmonary atresia with intact ventricular septum and intermediate right ventricle: early in-hospital and mediumterm outcomes. J Thorac Cardiovasc Surg 2011; 141: 1355 1361

[27] Daubeney PEF, Sharland GK, Cook AC et al. Pulmonary atresia with intact ventricular septum. Circulation 1998; 98 : $562-566$

[28] Glatz AC, Petit C], Goldstein BH et al. Comparison between patent ductus arteriosus stent and modified blalock-taussig shunt as palliation for infants with ductal-dependent pulmonary blood flow. Circulation 2018; 137: 589-601

[29] Schranz D, Bauer A, Reich B et al. Fifteen-year single center experience with the "giessen hybrid" approach for hypoplastic left heart and variants: current strategies and outcomes. Pediatr Cardiol 2014; 36: $365-373$
[30] Akintuerk H, Michel-Behnke I, Valeske K et al. Stenting of the arterial duct and banding of the pulmonary arteries. Circulation 2002; 105: 1099-1103

[31] Baba K, Kotani Y, Chetan D et al. Hybrid versus norwood strategies for single-ventricle palliation. Circulation 2012; 126: S123-S131

[32] Dave H, Rosser B, Knirsch W et al. Hybrid approach for hypoplastic left heart syndrome and its variants: the fate of the pulmonary arteries. Eur J Cardiothorac Surg 2014; 46 : $14-19$

[33] Hirata $\mathrm{Y}$, Miyata $\mathrm{H}$, Hirahara $\mathrm{N}$ et al. Long-term results of bilateral pulmonary artery banding versus primary Norwood procedure. Pediatr Cardiol 2018; 39: 111-119

[34] Karamlou T, Overman D, Hill KD et al. Stage 1 hybrid palliation for hypoplastic left heart syndrome-assessment of contemporary patterns of use: an analysis of The Society of Thoracic Surgeons Congenital Heart Surgery Database. JThorac Cardiovasc Surg 2015; 149: 195-201-202

[35] Feinstein JA, Benson DW, Dubin AM et al. Hypoplastic left heart syndrome: current considerations and expectations. J Am Coll Cardiol 2012; 59: S1 - S42

[36] Gossett JG, Rocchini AP, Lloyd TR et al. Catheter-based decompression of the left atrium in patients with hypoplastic left heart syndrome and restrictive atrial septum is safe and effective. Catheter Cardiovasc Interv 2006; 67: 619-624

[37] Hoque T, Richmond M, Vincent JA et al. Current outcomes of hypoplastic left heart syndrome with restrictive atrial septum: a single-center experience. Pediatr Cardiol 2013; 34: $1181-1189$

[38] Vlahos AP. Hypoplastic left heart syndrome with intact or highly restrictive atrial septum: outcome after neonatal transcatheter atrial septostomy. Circulation 2004; 109: $2326-2330$

[39] Chaturvedi RR, Ryan G, Seed M et al. Fetal stenting of the atrial septum: technique and initial results in cardiac lesions with left atrial hypertension. International J Cardiol 2013; 168: $2029-2036$

[40] Kalish BT, Tworetzky W, Benson CB et al. Technical challenges of atrial septal stent placement in fetuses with hypoplastic left heart syndrome and intact atrial septum. Catheter Cardiovasc Interv 2014; 84: 77-85

[41] Sathanandam S, Justino H, Waller BR et al. Initial clinical experience with the Medtronic Micro Vascular Plug ${ }^{\mathrm{TM}}$ in transcatheter occlusion of PDAs in extremely premature infants. Catheter Cardiovasc Interv 2017; 89: 1051 - 1058

[42] Morville P, Douchin S, Bouvaist $\mathrm{H}$ et al. Transcatheter occlusion of the patent ductus arteriosus in premature infants weighing less than $1200 \mathrm{~g}$. Arch Dis Childhood Fetal Neonat Edn 2018; 103: F198-F201

\section{Bibliografie}

DOI https://doi.org/10.1055/a-0747-8956

Neonatologie Scan 2019; 08: 187-203

(c) Georg Thieme Verlag KG Stuttgart · New York

ISSN 2194-5462 


\section{Punkte sammeln auf CME.thieme.de}

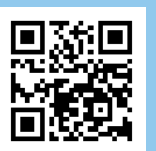

Diese Fortbildungseinheit ist in der Regel 12 Monate online für die Teilnahme verfügbar.

Den genauen Einsendeschluss finden Sie unter https://eref.thieme.de/CXBVBQE.

Sollten Sie Fragen zur Online-Teilnahme haben, finden Sie unter https://cme.thieme.de/hilfe

eine ausführliche Anleitung. Wir wünschen viel Erfolg beim Beantworten

der Fragen!

Unter https://eref.thieme.de/CXBVBQE oder über den QR-Code kommen Sie direkt zur Startseite des Wissenstests.

VNR 2760512019156640318

\section{Frage 1}

Bei welchem kritischen angeborenen Herzfehler kann die Differenzialdiagnose zur persistierenden pulmonalen Hypertension des Neugeborenen besonders schwierig sein?
A kritische Aortenklappenstenose
B d-Transposition der großen Arterien
C Pulmonalatresie mit intaktem Ventrikelseptum
D hypoplastisches Linksherzsyndrom
E totale Lungenvenenfehlmündung mit Obstruktion

\section{Frage 2}

Welches Gefäß wird nicht als Zugang für Herzkatheterinterventionen beim Neugeborenen verwendet?
A A. femoralis
B A. carotis
C A. radialis
D V. femoralis
E V. umbilicalis

\section{Frage 3}

Herzkatherinterventionen im Neugeborenenalter sind mit dem Risiko verschiedener Komplikationen verbunden. Welche gehört nicht dazu?
A Blutverlust
B strahleninduzierte akute Linsentrübung
C Schenkelblock
D AV-Block III
E arterielle Thrombose

\section{Frage 4}

Eine der Aussagen zur Ballonatrioseptostomie (BAS) nach Rashkind ist falsch. Welche?

A Die BAS ist die älteste Herzkatheterintervention beim Neugeborenen.

B Die BAS wird meist ultraschallgestützt - ohne Fluoroskopie - durchgeführt.

C Als Gefäßzugang wird die Umbilikal- oder eine der Femoralvenen verwendet.

D Jedes Neugeborenen mit d-Transposition der großen Arterien benötigt vor der Korrektur-OP eine BAS.

E Bei hochgradig restriktivem Vorhofseptum kann die BAS unmittelbar nach Geburt erforderlich sein.

\section{Frage 5}

Welche Aussage zur valvulären Pulmonalstenose trifft nicht zu?

A Die Pulmonalstenose kann so ausgeprägt sein, dass die pulmonale Perfusion ductusabhängig ist.

B Die Druckbelastung des rechten Ventrikels kann zu einer ausgeprägten Trikuspidalinsuffizienz führen.

C Ein Rechts-Links-Shunt auf Vorhofebene kommt nicht vor.

D Der Schweregrad der Stenose kann mittels invasiver Druckmessung bestätigt werden.

E Die Standardtherapie der isolierten valvulären Pulmonalstenose ist die Ballonvalvuloplastie. 


\section{Punkte sammeln auf CME.thieme.de}

Fortsetzung ...

\section{Frage 6}

Nur eine der folgenden Aussagen zur kritischen Aortenklappenstenose ist korrekt. Welche?

A Eine pränatale Diagnose ist heute Standard, da die Situation bei Einstellung des Vierkammerblicks eindeutig ist.

B Die klinische Diagnose ist zuverlässig über das stets vorhandene, typische, laute Systolikum über dem zweiten Interkostalraum rechts parasternal möglich.

C Die Erkrankung führt ausschließlich zu einer Druckbelastung des linken Ventrikels, sodass die Funktion der rechten Herzanteile nicht beeinträchtigt ist.

D Die Ballonvalvuloplastie der Aortenklappe ist nur über einen antegraden Zugang möglich.

E Nach Ballonvalvuloplastie der Aortenklappe kann es zu einer Insuffizienz der Klappe kommen.

\section{Frage 7}

Wodurch ist die Pulmonalatresie mit intaktem Ventrikelseptum gekennzeichnet?

A Die Erkrankung ist durch eine ausgesprochene Homogenität ihrer Ausprägung gekennzeichnet.

B Zur Bestätigung der Diagnose ist eine Kardio-MRT unverzichtbar.

C Eine Behandlung mit Prostaglandin $\mathrm{E}_{1}$ ist in der Regel nicht erforderlich, da die pulmonale Perfusion über aortopulmonale Kollateralen erfolgt.

D Eine katheterinterventionelle Therapie ist auch bei langstreckiger muskulärer Atresie möglich, da das atretische Segment nach Perforation und Rekanalisation mit einem ummantelten Stent überbrückt werden kann.

E In manchen Fällen entscheidet sich erst nach längerer Zeit, ob eine biventrikuläre Zirkulation möglich ist.

\section{Frage 8}

Welche Aussage zum hypoplastischen Linksherzsyndrom ist falsch?

A Die Diagnose kann bereits pränatal bei Darstellung eines Vierkammerblicks beim Fetus gestellt oder zumindest vermutet werden.

B Bei der pränatalen spezialisierten Feindiagnostik sollte unter anderem die Frage nach der Funktion der Vorhofkommunikation beantwortet werden.

C Die Prognose der Behandlung mittels univentrikulärer Palliation hat sich in den letzten Jahren erheblich verbessert.

D Nach rascher Herstellung einer ausreichenden Vorhofkommunikation unterscheidet sich die Prognose der Patienten mit hochgradig restriktivem Foramen ovale nicht mehr von denen mit primär ausreichend großer Vorhofkommunikation.

E Langfristig muss mit einer erhöhten Morbidität gerechnet werden.

\section{Frage 9}

Welche Aussage zur Aortenisthmusstenose (ISTA) ist falsch?

A In der Diagnostik der ISTA hat die wiederholte vergleichende Pulspalpation an oberen und unteren Extremitäten an Bedeutung verloren, da die Diagnose in der Regel pränatal gestellt wird.

B Die ISTA tritt isoliert und in Kombination mit anderen Herzfehlern auf.

C Auch nach genauer pränataler Diagnosestellung einer ISTA ist postnatal eine Wiederholung der Echokardiografie zur Therapieplanung erforderlich.

D Die Behandlung der ISTA im Neugeborenenalter erfolgt primär chirurgisch.

E In ausgewählten Fällen kann eine präoperative Stabilisierung mit einer katheterinterventionellen Therapie (Ballondilatation des Aortenisthmus) sinnvoll sein.

\section{Frage 10}

Welcher Eingriff ist keine typische Herzkatheterintervention im Neugeborenenalter?

A Vergrößerung eines Vorhofseptumdefektes

B Ballondilatation von Klappen

C Schaffung eines Vorhofseptumdefektes

D Verschluss von Gefäßen

E transluminaler Klappenersatz 